\title{
Influence of Narrative Elements on User Behaviour in Photorealistic Social VR
}

\author{
Anonymous Author(s)
}
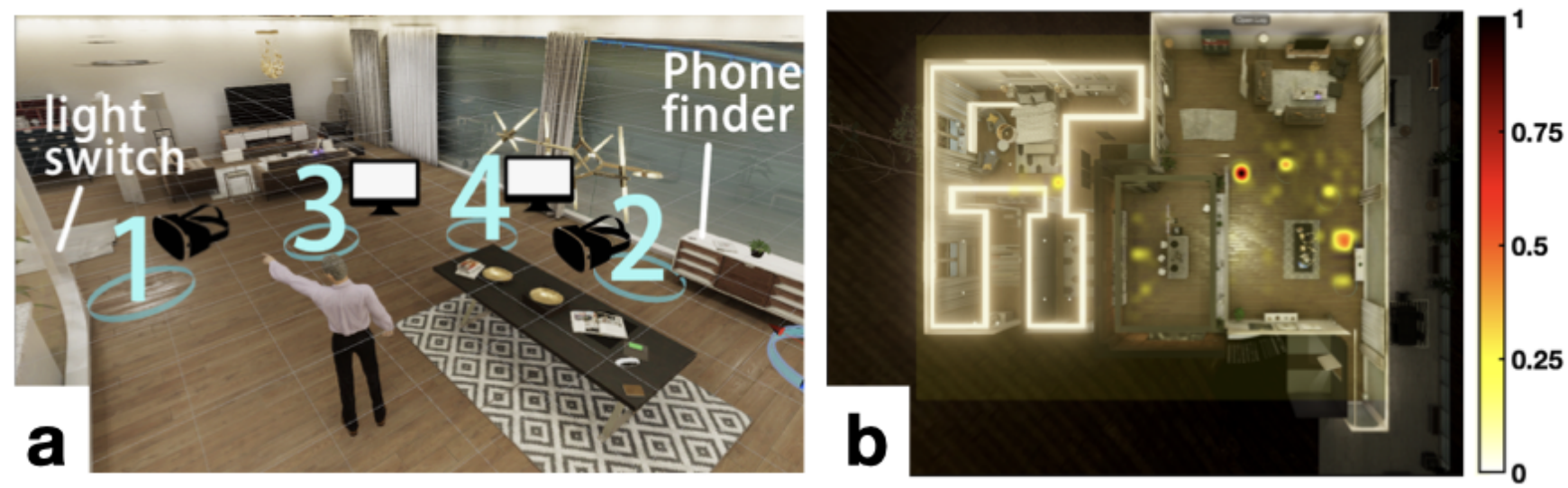

Figure 1: a) Living room where the starting position of each user is indicated. In details, 1 and 2 are HMD users while users in position 3 and 4 are desktop users. There are also two interactive objects (i.e., the light switch on the left and phone finder on the right) and the main virtual character, detective Sarge. b) A floor map of the virtual house is reported with the user heatmap of main locations visited over time.

\begin{abstract}
Social Virtual Reality (VR) applications are becoming the next big revolution in the field of remote communication. Social VR provides the possibility for participants to explore and interact with a virtual environments and objects, feelings of a full sense of immersion, and being together. Understanding how user behaviour is influenced by the shared virtual space and its elements becomes the key to design and optimize novel immersive experiences that take into account the interaction between users and virtual objects. This paper presents a behavioural analysis of user navigation trajectories in a 6 degrees of freedom, social VR movie. We analysed 48 user trajectories from a photorealistic telepresence experiment, in which subjects experience watching a crime movie together in VR. We investigate how users are affected by salient agents (i.e., virtual characters) and by the narrative elements of the VR movie (i.e., dialogues versus interactive part). We complete our assessment by conducting a statistical analysis on the collected data. Results indicate that user behaviour is affected by different narrative and interactive elements. We present our observations, and we draw conclusions on future paths for social VR experiences.
\end{abstract}

Permission to make digital or hard copies of all or part of this work for personal or classroom use is granted without fee provided that copies are not made or distributed for profit or commercial advantage and that copies bear this notice and the full citation on the first page. Copyrights for components of this work owned by others than ACM must be honored. Abstracting with credit is permitted. To copy otherwise, or republish, to post on servers or to redistribute to lists, requires prior specific permission and/or a fee. Request permissions from permissions@acm.org.

MMVE'21, September 28, 2021, Istanbul, Turkey

(c) 2018 Association for Computing Machinery.

ACM ISBN 978-1-4503-XXXX-X/18/06 .. \$15.00

https://doi.org/10.1145/1122445.1122456

\section{CCS CONCEPTS}

- Information systems $\rightarrow$ Multimedia streaming; • Humancentered computing $\rightarrow$ Virtual reality; User studies.

\section{KEYWORDS}

User Analysis, 6-DOF, Social Virtual Reality, Point Cloud, Immersive Movie

\section{ACM Reference Format:}

Anonymous Author(s). 2018. Influence of Narrative Elements on User Behaviour in Photorealistic Social VR. In International Workshop on Immersive Mixed and Virtual Environment Systems (MMVE'21), September 28, 2021, Istanbul, Turkey. ACM, New York, NY, USA, 6 pages. https://doi.org/10.1145/ 1122445.1122456

\section{INTRODUCTION}

Immersive Virtual Reality (VR) applications are going through a rapid evolution of technology, getting integrated in daily-life devices such as smartphones and laptops. Therefore, it is possible to imagine that in the near future video calls will give users a completely different experience than now: people will be able to chat, walk together, interact with virtual objects and watch events such as concerts or movies together in a common virtual environment [15]. This is what social $V R$ applications are promising to enable, becoming the next big revolution in the field of communications. VR applications are already going beyond the passive media consumption of traditional video allowing for a higher degree of immersiveness and interaction by placing the users at the centre of the action; a further step forward is attempted by social VR. This emerging remote communication tool is indeed aiming at 
overstepping current remote communications through 2D screens, enabling instead virtual co-presence of more users within the same virtual environment and allowing body interactions similarly to face-to-face communication $[7,13,14]$. The new challenge is therefore to increase the realism in the virtual experience and interaction. Therefore, the key aspect that needs to be fully understood in order to advance in this technology is the user, the main director of the virtual experience.

Emerging Social VR platform, such as Facebook Horizon ${ }^{1}$ and Mozilla Hubs ${ }^{2}$, have rapidly grown in popularity. In most of these applications, participants take part of 3D virtual spaces through a computer-generated and customised avatar, and they can interact with each other. Physical displacement and proxemic interactions in virtual environments have been analysed to investigate which social cues are the most influencing and therefore are needed to ensure presence and immersion [12,26]. Moreover, many works have investigated the advantages to have a more realistic self-representation versus a 3D avatar providing a higher degree of immersion and presence in the VR experience [8,11]. Therefore recently, a more natural self-representation of participants has been introduced, thanks to real-time acquisitions and reconstructions of point clouds by depth cameras [9]. Not to be neglected is the technological aspect of these new applications. Social interactions and photorealistic representations come with both computational and bandwidth overhead for transmission and rendering [20]. Understanding user behaviour represents the first step towards the building of real-time and realistic VR systems at large that can optimize delivery based on user-centred adaptation [23, 25].

In this paper we focus on a better understanding of how people interact with a virtual environment and other users within it. In details, we present a first attempt of behavioural analysis in a 6-Degrees-of-Freedom (DoF) social and interactive VR movie. We based our analysis on navigation trajectories collected in a novel type of interactivity crime movie: four users, either equipped by a head-mounted display (HMD) or desktop computer and a controller, were watching together a VR crime solving movie [6]. Figure 1 (a) show a snapshot of the living room in which the story mainly takes place, with its main virtual character. The challenges that we aim to address in our analysis are twofold. As first we need to consider both the new physical settings and locomotion functionalities given to users in a 6-DoF system. The user now not only can select the portion to be displayed by rotating the head as in a 3-DoF system but can also move inside the virtual environment changing the distance and perspective with the displayed content. The second aspect to consider are the new social and interactivity features of the application that brought an added level of dynamics. For instance, during the experience participants are asked to make simple tasks, such as to look for and to press a bottom; their action influences directly the narration of the story since the time to solve the task is not fixed. Therefore, we compare user behaviour in terms of spatial displacements in the virtual environment and viewing direction with respect to movie characters and other participant within the whole experience. In particular, we show how much narrative

\footnotetext{
${ }^{1}$ https://www.oculus.com/facebook-horizon/

${ }^{2}$ https://hubs.mozilla.com
}

elements of the movie, such as virtual characters movements or request of interactions, influence user behaviour.

\section{RELATED WORK}

Depending on the locomotion functionalities and the type of content representation, navigation in immersive media environments can range from 3-DoF to 6-DoF. In the first case, users are placed at the center of a spherical content, and by changing their viewing angle, they are able to select the portion of the content they want to visualize at any given moment. In case of 6-DoF, users are additionally able to change their position in the $3 \mathrm{D}$ environment, which is now populated with volumetric objects that can be observed from any position and viewing angle. The 6-DoF scenario promises a much more natural interaction and exploration of the 3D scene than its 6-DoF counterpart, thus increasing the feeling of presence and immersiveness [2] and opening the gate to VR innovations. Incorporating information about user behaviour in user-centric systems is of paramount importance to optimize transmission and rendering $[18,25]$, highlighting the need to understand users' behaviour in 3- and 6-DoF VR settings. In the first scenario, users trajectories has been intensely analysed by different tools: angular velocity, frequency of fixation, and mean exploration angles, saliency maps $[4,21]$ but also through clustering approach and information theory metrics $[16,17,19]$. In the past, preliminary study on locomotion and display technology were presented for CAVE environments $[3,24]$ and more recently $6-\mathrm{DoF}$ trajectories have been analysed in terms of angular velocity [1,22]. Despite the great potentials, the study of user behaviour has been overlooked focusing only on a general characterisation and none specific tool or procedure has been proposed for 6-DoF trajectories.

\section{A SOCIAL VR MURDER MYSTERY MOVIE}

We contextualize our experiment in a social VR setting, in which 4 users to experience an immersive movie, occasionally being called to participate and offering their input. The scenario allows for 6DoF navigation within a photorealistic $3 \mathrm{D}$ environment, which is populated by three virtual characters. In the following, we describe the movie timeline, the setup used during experiments and finally, we show some general performance of the system.

\subsection{Movie plot}

The interactive and immersive VR movie used in the experiments is about a murder mystery investigation [6]. The victim is Elena Armova, who lived in a luxury apartment in central London. A total of 4 participants are selected to aid the investigations lead by detective Sarge Hoffsteler and his assistant, Rachel Tyrell. The investigation is split into 3 chapters, as depicted in Figure 2. In chapter 1 , the 4 users are placed in the virtual living room of the victim Ms. Armova, adopting an initial fixed positions indicated in Figure 1 (a). Participants mainly listen to the victim interrogation, which is possible thank to a futuristic machine based on artificial intelligence. There are also two moments in which users are asked to interactively interact with objects in the scene:

- user 1 is asked to switch on the light,

- user 2 has to pick up a phone finder controller and press the bottom. 


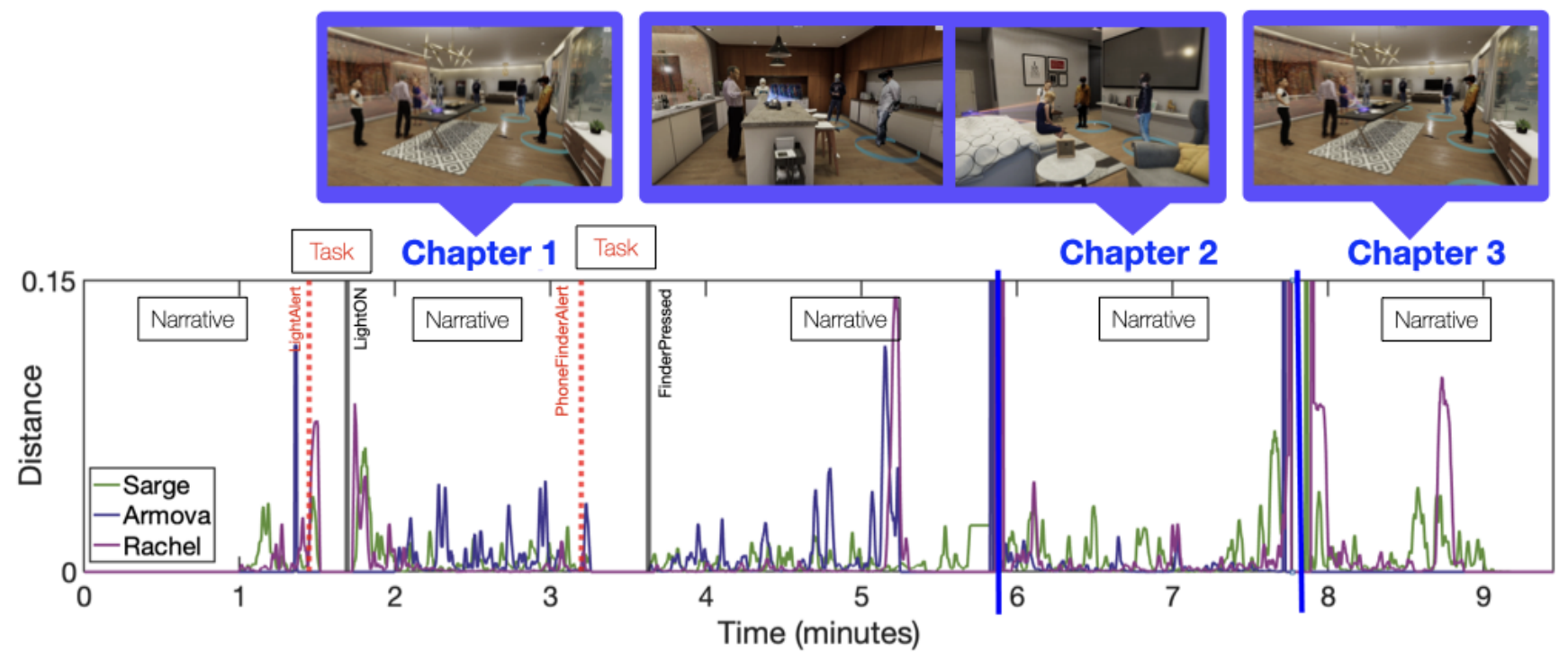

Figure 2: Timeline of the VR murder mystery movie: and spatial movements of virtual characters over time. A screenshot per each chapter is reported.

Therefore, we can distinguish two moments with a task for participants and three narrative moments where mainly virtual characters are talking and walking around the scene, respectively named task and narrative in Figure 2. At the end of chapter 1, users are split into two groups: user 1 and 3 are conducted in the virtual kitchen with detective Sarge, whereas user 2 and 4 are led to the virtual bedroom with Rachel and Ms. Armova. In both the room, participants are listening to narrative dialogues of virtual characters. At the end of chapter 2, the users are brought back together in the virtual living room for the final chapter, where detective Sarge describes how the murder has been solved.

\subsection{Experimental Setup}

A low-latency volumetric video delivery pipeline, based on point cloud representation, was used to place each participant into the virtual scene [9]. Each user was captured using 3 Kinect Azure devices, placed in a circle around them, $120^{\circ}$ apart from each other. This allowed each participant to be captured from multiple angles, ensuring a photorealistic representation while they interacted with the scene.

Two devices were used to visualize the social VR experience: users 1 and 2 were equipped with Oculus Rift HMDs, complete with controllers, whereas users 3 and 4 could watch the scene through 50 -inch monitors, and could navigate using gaming joysticks. For HMD users, teleportation was enabled in key locations of the scene, as physical locomotion was restricted due to the acquisition setup, whereas for desktop users, movement was enable through the gaming joystick. Due to the configuration of the controllers, only HMD users were able to engage with the interactive elements in the scene.

A total of 48 participants was recruited for the experiment, resulting in 12 social VR sessions. The participants were between 21 and 56 years old ( $\mu=34.9, \sigma=10.3)$. The gender distribution was balanced (23 males, 25 females). All of them were fluent in English, and had no motor or visual impairment.
During the sessions, the position and rotation of the camera objects associated with each users were recorded at $30 \mathrm{~Hz}$. Moreover, timestamped data was logged in a machine readable format, after we determined that this log collection had no discernible influence on system performance. The logs also contained enough information to allow combining the data from the individual machines after the session with the correct timestamp information (with an uncertainty of network roundtrip time, which was good enough for the problem at hand). Logged information included number of decoded points, transmission delay, and framerate. From the logged data it was possible to compute, for each rendering device, the elapsed time between the encoding of a given frame of the point cloud representation and its rendering on the virtual scene. This information effectively provided the latency between encoding and rendering, for each device under use. The data was obtained at the granularity of one second, to avoid disrupting the performance of the system. The observed latency in all sessions was generally lower than 1 second, and was remarkably smaller for self-representation. In particular, mean latency for self-representation was 0.1147 seconds (25 percentile: 0.079 ; median: $0.105 ; 75$ percentile: 0.136 ), whereas for the rest of the cases, it amounted to 0.5526 seconds ( 25 percentile: 0.408 ; median: $0.538 ; 75$ percentile: 0.689 ). On average, the point count for each frame was 86342 points per cloud (25 percentile: 82412.5 ; median: 90268 ; 75 percentile: 95727.5 ). Finally, the observed framerate for all the representations was on average 9.08 frames per second (25 percentile: 7.5 ; median: 8.9 ; 75 percentile: 10.6).

\section{BEHAVIOURAL ANALYSIS}

A general overview of the exploration behaviour of users is given in Figure 1 (b), which shows a heatmap of the most visited locations in the virtual house, obtained by aggregating all the position data collected in the experiment. As described in Section 3.1, large part of the movie takes place in the living room (Chapter 1 and 3), which is 


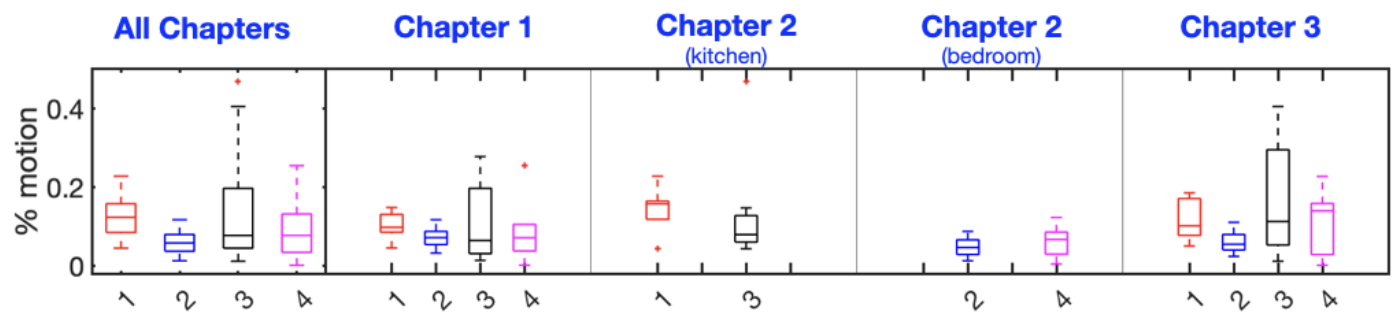

Figure 3: User's motion based on both spatial and rotation movements per each session in the movie.

reflected in the figure. The most visited locations correspond to the initial positions of user 1 and 2, due to their movement restriction as HMD users (i.e., only teleportation in fixed locations was allowed). More generally, the initial positions of all users are clearly visible in the heatmap; additional yellow spots outside of the predefined circles indicate most likely regions to be visited by desktop users. Whereas movement is more spread in the living room, in the other smaller room of the house, kitchen and bedroom, it appears much more spatially focused, indicating that participants were more static in these spaces.

Figure 3 displays the boxplot comparison between percentage of motion exhibited by each user. For every frame, we considered the user to be "in motion" if either their relative position with respect to the previous frame changed more than $0.05 \mathrm{~cm}$, or if any of their rotation angles varied by more than $0.01 \mathrm{rad}\left(0.573^{\circ}\right)$. Both measures were taken into account to cover both spatial exploration behavior, as well as changes in viewing angles. The percentage was then computed with respect to the total number of frames. It can be observed that desktop users (user 3 and 4) exhibit a larger percentage of motion over the course of the movie, with respect to HMD users. Motion was present in the first and last chapter with wider distributions, indicating larger variance in the way users behaved, whereas in the second chapter, users generally showed smaller variance in percentage of motion.

In order to better understand how users behaviour changed over time as the movie progressed, we analyse their position and orientation with respect to the virtual characters (avatars), namely Sarge, Rachel and Armova. To give an idea about avatar displacements and actions in the movie, Figure 2 shows distance covered by each character over time. We can notice that during task parts, avatars are statically waiting for users to make the action. The characters of Armova and Rachel are leaving the scene of chapter 1 before Sarge, and indeed around the 5-minute mark, there are two peaks in their displacement. It is worth noting that Armova is in the scene only in chapter 1 and 2. Figure 4 shows the distribution of relative position and orientation of the users with respect to the avatars, separately per each chapter of the movie. In details, the first line of each subplot depicts the distance between each user and each avatar. The second line of subplots shows instead the angle between user's viewing direction and the vector which connect user and avatar at any given time. This angle indicates therefore if user is looking towards direction of character location $(\theta \rightarrow 0)$ or in in the opposite direction $(\theta \rightarrow \pi)$. In chapter 1 (Figure $4(\mathrm{a})$ ), it is therefore interesting to notice the different user's behaviour between narrative and task parts of the story. During the three narrative parts, users tend to further explore the environment around them: even if on average, users are looking in the direction of the three virtual characters, their variance indicates a non-uniform behaviour over time, suggesting that participants where also looking around. In the two task parts, instead, the distribution of both spatial distance and angle values is narrower. In particular, for the first task, a wider distribution in terms of viewing angle, which skews further away from the avatar, can be observed for user 1, which is the one tasked with pressing the button to turn on the light. This difference might be due to the mismatching of difficulty between the two task. Indeed, participants took on average around 13.33 seconds to switch on the light, while picking up the phone finder controller and press the button required around 25.58 seconds. To validate our intuition, we perform a non-parametric Mann-Whitney statistical test between the distance and angle to avatar recorded during the narrative parts of chapter 1 , and the task parts. To avoid bias induced by the large number of samples, we performed random sampling on the data, selecting $\mathrm{N}=200$ samples across the distance vector, and repeating the procedure across 200 sampling runs. We used Fisher's method $[5,10]$ to combine the probabilities, obtaining that the type of task has a significant effect on the distance $\left(\chi^{2}=3202.5\right.$, $p<.001)$ and on the viewing angle $\left(\chi^{2}=4771.8, p<.001\right)$. In fact, distance to avatar appears to be statistically different between all sub-parts of chapter 1, indicating varying behavior in terms of spatial movements between as the time progressed. In terms of viewing angle, however, no discernible effect is observed on different narrative parts with respect to the viewing angle (narr.1 - narr.2: $\chi^{2}=407.5, p=0.387$; narr. 1 - narr. $3: \chi^{2}=407.5, p=0.388$; narr. 2 - narr.3: $\left.\chi^{2}=421.5, p=0.221\right)$, whereas the two tasks exhibited significantly different viewing angle distributions $\left(\chi^{2}=1270.6\right.$, $p<.001$ ). In chapter 2 (Figure 4 (b)) participants are moved in different rooms, kitchen and bedroom, both of them smaller compare to the initial living room. This different ambient dimension affects indeed user's behaviour: participants in general are much more static compare to the previous chapter. As last observation, we notice that users in chapter 3 (Figure 4 (c)) behave similar to narrative moments of chapter 1 : there are exploration movements both in terms of spatial and angle values.

Finally, we also analyse the user's position and viewing direction with the respect to other participants. As in the previous analysis, Figure 5 depicts the spatial distance and angular difference between each couple of users per each chapter of the movie. While in the previous comparison between user and avatars, the behaviour of the latter was known and stayed constant for each experiment, in this case both users under exam have varying positions and viewing directions over time. However, some general behaviour can be extracted also under these conditions confirming the previous 


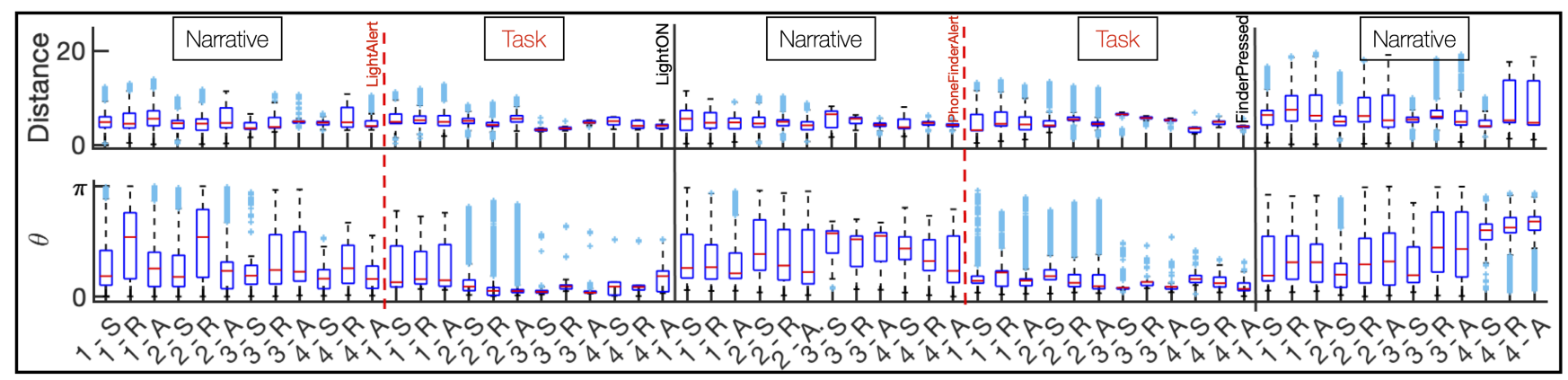

(a) Chapter 1

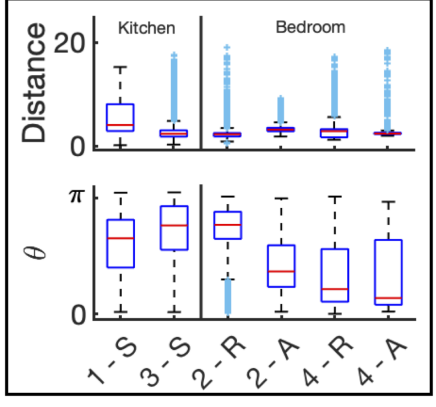

(b) Chapter 2

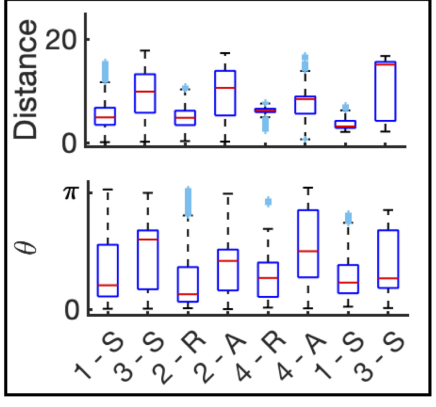

(c) Chapter 3

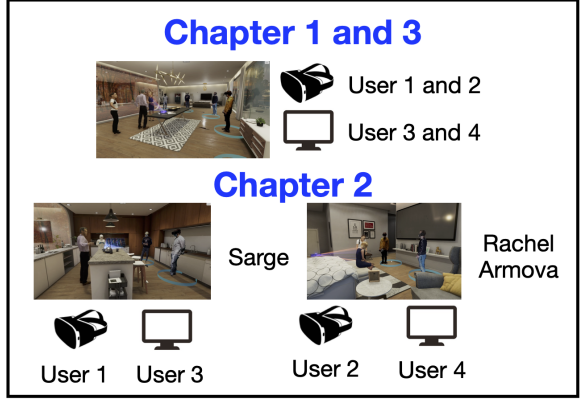

(d) Legend

Figure 4: Spatial distance (first line of each subplot) and angle (second line of each subplot) between users and avatars per each chapter of the storytelling.

findings. For instance, during task parts in chapter 1 (Figure 5 (a)), the distribution of angle values is quite narrow for most of the couples, indicating that they moved their attention from the avatars to another participant. On the contrary, during narrative parts, these distributions are wider highlighting that users were not fixating on each other, rather exploring the scene and the virtual characters within. Statistical tests show that the effect of the task is significant on the viewing angle $\left(\chi^{2}=2465.6, p<.001\right)$, and differences among narrative and task sub-parts of chapter 1 are always significant $(p<.001)$, with the exception of the first and the last part of chapter $1\left(\chi^{2}=415.9, p=0.282\right)$. In terms of spatial displacements, the distance between users remains low in all the chapters of the movie. The effect of task versus narrative is significant $\left(\chi^{2}=1270.4\right.$, $p<.001)$, and distance always differs significantly between subparts $(p<.001)$, with the exception of the first two narrative parts $\left(\chi^{2}=418.8, p=0.249\right)$.

In summary, the behavioural analysis we conducted of users in a social VR movie leads to the following observations:

- Observation 1: during narrative moments of the story, participants are more inclined to explore the virtual environment with general attention to virtual characters;

- Observation 2: the request of interactions with the content by a specific user (e.g., to press a bottom) leads to reduced movement, while the attention is more focused on the task or on other participants;

- Observation 3: the size of the virtual environment in which is located the experience also affect the user's behaviour. In particular, large rooms seem to be more conductive of exploratory behavior, whereas in smaller rooms, less variation in position or viewing angle is observed.

\section{CONCLUSION}

In this paper, we analysed the user's behaviour during a photorealistic telepresence experiment developed on a volumetric social VR system. We mainly investigated the influence of narrative elements of the story, such as dialogues or interactive task, on participants' movements. Our results show indeed that the motion during the VR experience was affected by the storytelling. More static and focused behaviour happened when a task (either to switch on the light or press a bottom in a controller) was requested to be done by a specific participant. On the contrary, exploration movements were more frequent when virtual characters were talking in the scene. These observations are key factors to be further investigated, in order to develop immersive and interactive applications. Further work is needed to understand the effect of storytelling elements and cues on user behaviour, in order to design social VR experiences that can effectively be optimized around the users.

\section{REFERENCES}

[1] E. Alexiou, N. Yang, and T. Ebrahimi. 2020. PointXR: A toolbox for visualization and subjective evaluation of point clouds in virtual reality. (2020).

[2] F. Buttussi and L. Chittaro. 2017. Effects of different types of virtual reality display on presence and learning in a safety training scenario. IEEE transactions on visualization and computer graphics (2017).

[3] C. Christou, A. Tzanavari, K. Herakleous, and C. Poullis. 2016. Navigation in virtual reality: Comparison of gaze-directed and pointing motion control. In 18th mediterranean electrotechnical conference. 


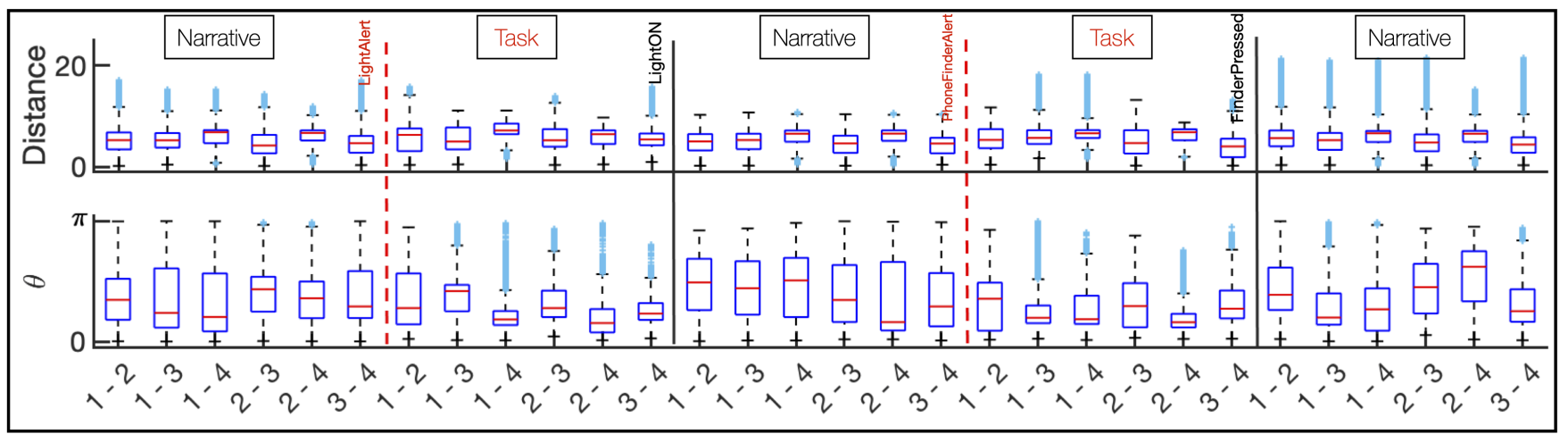

(a) Chapter 1

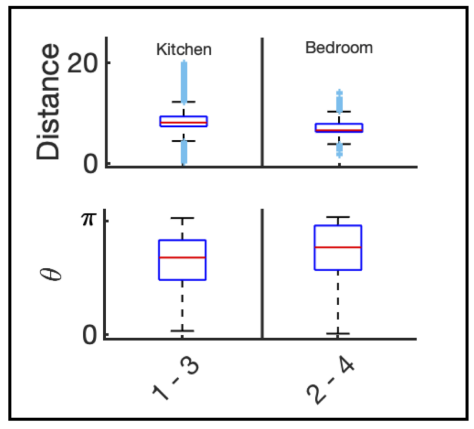

(b) Chapter 2

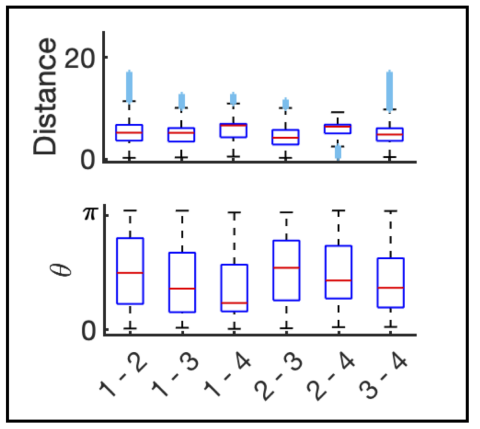

(c) Chapter 3

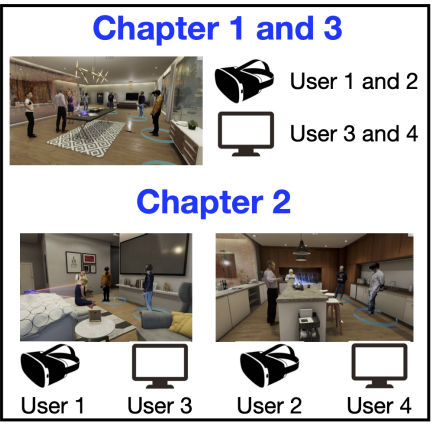

(d) Legend

Figure 5: Spatial distance (first line of each subplot) and angle (second line of each subplot) between couples of users per each chapter of the storytelling.

[4] X. Corbillon, F. De Simone, and G. Simon. 2017. 360-degree video head movement dataset. (2017)

[5] Ronald Aylmer Fisher et al. 1934. Statistical methods for research workers. Statistical methods for research workers. 5th Ed (1934)

[6] Anonymized for review. 2021. A Collaborative VR Murder Mystery using Photorealistic User Representation. In (Submitted to) the IEEE Virtual Reality Conference.

[7] G. Freeman and D. Maloney. 2021. Body, Avatar, and Me: The Presentation and Perception of Self in Social Virtual Reality. (2021).

[8] G. Gamelin, A. Chellali, S. Cheikh, A. Ricca, C. Dumas, and S. Otmane. 2020 Point-cloud avatars to improve spatial communication in immersive collaborative virtual environments. Personal and Ubiquitous Computing (2020).

[9] J. Jansen, S. Subramanyam, R. Bouqueau, G. Cernigliaro, M. M. Cabré, F. Pérez, and P. Cesar. 2020. A Pipeline for Multiparty Volumetric Video Conferencing: Transmission of Point Clouds over Low Latency DASH. In Proceedings of the 11th ACM Multimedia Systems Conference.

[10] James T Kost and Michael P McDermott. 2002. Combining dependent P-values. Statistics \& Probability Letters 60, 2 (2002), 183-190.

[11] M. E. Latoschik, D. Roth, D. Gall, J. Achenbach, T. Waltemate, and M. Botsch. 2017. The effect of avatar realism in immersive social virtual realities. In Proceedings of the 23rd ACM Symposium on Virtual Reality Software and Technology.

[12] D. A. Le, B. MacIntyre, and J. Outlaw. 2020. Enhancing the Experience of Virtual Conferences in Social Virtual Environments. In 2020 IEEE Conference on Virtual Reality and 3D User Interfaces Abstracts and Workshops (VRW). IEEE, 485-494.

[13] J. Li, V. Vinayagamoorthy, R. Schwartz, W. IJsselsteijn, D. A. Shamma, and P. Cesar 2020. Social VR: A New Medium for Remote Communication and Collaboration. In Extended Abstracts of the 2020 ACM CHI Conference on Human Factors in Computing Systems.

[14] J. McVeigh-Schultz, A. Kolesnichenko, and K. Isbister. 2019. Shaping Pro-Social Interaction in VR: An Emerging Design Framework. In Proceedings of the 2019 ACM CHI Conference on Human Factors in Computing Systems.

[15] F. Moustafa and A. Steed. 2018. A longitudinal study of small group interaction in social virtual reality. In Proceedings of the 24th ACM Symposium on Virtual Reality Software and Technology.

[16] A. T. Nasrabadi, A. Samiei, A. Mahzari, R. P. McMahan, R. Prakash, M. C. Q. Farias, and M. M. Carvalho. 2019. A Taxonomy and Dataset for $360^{\circ}$ Videos. Association for Computing Machinery.

[17] S. Rossi, F. De Simone, P. Frossard, and L. Toni. 2019. Spherical clustering of users navigating 360 content. In IEEE International Conference on Acoustics, Speech and Signal Processing.

[18] S. Rossi, C. Ozcinar, A. Smolic, and L. Toni. 2020. Do Users Behave Similarly in VR? Investigation of the User Influence on the System Design. ACM Transactions on Multimedia Computing, Communications, and Applications (TOMM) (2020).

[19] S. Rossi and L. Toni. 2020. Understanding user navigation in immersive experience: an information-theoretic analysis. In Proceedings of the 12th ACM International Workshop on Immersive Mixed and Virtual Environment Systems.

[20] S. Schwarz, M. Preda, V. Baroncini, M. Budagavi, P. Cesar, P. A. Chou, R. A. Cohen, M. Krivokuća, S. Lasserre, Z. Li, et al. 2018. Emerging MPEG standards for point cloud compression. IEEE Journal on Emerging and Selected Topics in Circuits and Systems (2018)

[21] V. Sitzmann, A. Serrano, A. Pavel, M. Agrawala, D. Gutierrez, B. Masia, and G. Wetzstein. 2018. Saliency in VR: How Do People Explore Virtual Environments?. In IEEE Transactions on Visualization and Computer Graphics.

[22] S. Subramanyam, J. Li, I. Viola, and P. Cesar. 2020. Comparing the Quality of Highly Realistic Digital Humans in 3DoF and 6DoF: A Volumetric Video Case Study. In IEEE Conference on Virtual Reality and 3D User Interfaces.

[23] S. Subramanyam, I. Viola, A. Hanjalic, and P. Cesar. 2020. User Centered Adaptive Streaming of Dynamic Point Clouds with Low Complexity Tiling. In Proceedings of the 28th ACM International Conference on Multimedia.

[24] C. Swindells, B. A Po, I. Hajshirmohammadi, B. Corrie, J. Dill, B. Fisher, and K. Booth. 2004. Comparing CAVE, wall, and desktop displays for navigation and wayfinding in complex 3D models. In IEEE Proceedings Computer Graphics International.

[25] J. Van der Hooft, T. Wauters, F. De Turck, C. Timmerer, and H. Hellwagner. 2019. Towards 6-DoF HTTP adaptive streaming through point cloud compression. In Proceedings of the 27th ACM International Conference on Multimedia.

[26] J. Williamson, J. Li, V. Vinayagamoorthy, D. A Shamma, and P. Cesar. 2021. Proxemics and Social Interactions in an Instrumented Virtual Reality Workshop. arXiv preprint arXiv:2101.05300 (2021). 